\title{
D'une approche globale vers une approche semi-distribuée en modélisation pluie-débit
}

\section{From a global to a semi-distributed approach in rainfall-runoff modelling}

\author{
par C. Loumagne, C. Michel, B. Palagos \\ Cemagref, Antony \\ J-C. Baudez \\ Cemagref, Varennes sur Allier \\ F. Bartoli \\ CNRS-CPB, Vandoeuvre les Nancy
}

The question whether a distributed approach is more relevant than a lumped one for rainfall-rumoff modelling has not yet been answered clearly. Most of the time, the problem is solved rather intuitively on a case by case basis. In this research, we attempted to give an objective answer to this problem, in order to progress in catchment modelling. Two complementary approaches were used.

In the first approach, we used a sample of 15 gauged catchment triplets. We tried to assess whether it was interesting or not to partition a catchment in two subunits during the modelling process. The goodness of fit, as expressed by the Nash and Sutcliffe criterion, did not show a significant difference between both modelling approaches.

In the second approach, we used the same catchment sample and measured, for each subunit, physical characteristics that we assumed relevant for its hydrological behaviour. Differences observed in the subunits characteristics were quantified to obtain an index measuring the potential interest of distributed modelling.

Both approaches gave indications about the interest of subcatchment. Therefore a comparison of results has been achieved in order to determine if it was possible to establish a link between the degree of heterogeneity and the success of a modelling type.

This comparison has showed a relation between the studied features and results of the two types of modelling, global and semi-distributed. The choice of a modelling type is linked to the size of basins when these are classified by family size and to the degree of heterogeneity.

However, the reduced size of the sample doesn't allow us to bring about a definitive answer to this question. Other researches must be undertaken to extend the study to more catchments.

\section{INTRODUCTION}

Pour identifier les conditions optimales d'aménagement et de gestion de la ressource en eau, et pour aborder la prévision des impacts, tant des phénomènes naturels que des actions anthropiques, il faut pouvoir analyser les processus majeurs à différentes échelles spatio-temporelles et savoir les modéliser.

Or, les facteurs déterminants dans les mécanismes hydrologiques sont extrêmement variables dans l'espace comme dans le temps, ce qui rend très complexe leur représentation mathématique.

Jusqu'à présent, seule une modélisation globale des processus avec des paramètres intégrant les hétérogénéités locales avait permis de proposer des outils simples et opérationnels pour la gestion et la prévision de la ressource en eau à l'échelle d'un bassin $[1,2,3]$.

Mais un des objectifs de l'hydrologie est d'obtenir des méthodes transposables d'un site à un autre ou d'une échelle à une autre. Pour ce faire, il est nécessaire de relier les paramètres du modèle à des grandeurs caractéristiques du milieu physique.

Aussi, pour tenir compte de l'hétérogénéité d'un bassin, une démarche assez souvent suivie est la discrétisation du milieu étudié avec une taille de maille telle que les paramètres physiques y sont supposés homogènes. En outre, la variabilité entre les mailles doit reproduire une distribution observée ou présumée des grandeurs physiques et de leurs 
dépendances par rapport à des grandeurs macroscopiques établies sur quelques points de mesures. Cette approche a suscité de nombreuses études qui ont permis de mieux appréhender les processus à l'échelle de la maille choisie mais s'est révélée peu satisfaisante quant aux améliorations apportées aux méthodes de gestion et de prévision opérationnelles $[4,5,6]$.

La problématique à résoudre est de pouvoir répondre à la question suivante non encore résolue : " L'approche discrétisée apporte-t-elle une amélioration par rapport à l'approche globale, pour la gestion et la prévision opérationnelle des débits? " Pour répondre le plus rigoureusement possible à cette question, nous avons, dans un premier temps, comparé les deux approches à l'aide d'un même modèle, afin de quantifier le gain de précision. Dans un second temps, indépendamment du reste, nous avons cherché à classer les bassins étudiés en fonction de leur hétérogénéité. Enfin, la mise en parallèle de ces deux comparaisons a permis d'étudier le lien de cause à effet reliant la variabilité entre les mailles et l'intérêt de discrétiser le milieu modélisé.

\section{II $\square$ COMPARAISON DE L'APPROCHE GLOBALE ET DE L'APPROCHE SEMI-DISTRIBUÉE}

Lorsqu'un certain cadre géographique doit être représenté dans un modèle, deux options sont possibles :

- quand on peut estimer que les variations spatiales des paramètres et des variables d'entrées peuvent être prises en compte d'une façon globale et qu'il n'y a pas lieu d'effectuer un découpage dans l'espace, on dit que le modèle est global,

- au contraire, quand l'étendue ou l'hétérogénéité du cadre physique, comme la concentration et la diversité spatiale des interventions anthropiques sur le système, peuvent conduire à préférer a priori une discrétisation de l'espace des entrées et des paramètres, on construit alors un modèle distribué. La forme des unités élémentaires qui seront représentées par un modèle global peut être arbitraire, par exemple des mailles carrées, ou fondée sur des divisions naturelles comme un découpage par sous-bassins. Par construction, un modèle distribué est un assemblage de sous-modèles globaux appliqués sur des mailles plus ou moins fines. Sur chacune des mailles, les variables spatiales et autres flux sont représentées par des scalaires. La globalisation au niveau d'une maille semble a priori d'autant plus légitime que la maille est plus petite, car les processus y opérant sont moins divers. Ainsi, l'utilisation de paramètres réels à l'échelle de la maille suppose implicitement que cette maille est homogène. Cependant, on introduit un besoin de modélisation supplémentaire qui est le modèle d'agrégation des flux en sortie des mailles constitutives.

Certains modèles en hydrologie essaient de prendre en compte l'hétérogénéité de l'occupation du sol dans les bassins versants en les découpant en parcelles fictives correspondant chacune à un type d'occupation, ces niveaux de découpage n'ayant pas un ordre hiérarchique les uns par rapport aux autres. Ces modèles sont appelés semi-distribués.

Ainsi pour prendre en compte les phénomènes hydrologiques variant spatialement, nous devions disposer d'un modèle distribué. Or, notre objectif n'était pas de comparer un modèle global $\mathrm{X}$ avec un modèle distribué $\mathrm{Y}$ différent, mais d'apprécier l'intérêt de découper le bassin versant en mailles plus petites pour mieux en modéliser les processus.
Par conséquent, et afin de n'introduire aucun biais relatif à la qualité des modèles, la comparaison entre les deux approches s'est articulée autour d'un même modèle dans deux configurations différentes, la globale et la distribuée, et ceci afin de pouvoir apporter une réponse quant à l'intérêt ou non de découper les bassins versants en fonction de leurs caractéristiques physiques.

Jusqu'à présent nous avions travaillé dans le domaine de la modélisation pluie-débit avec des modèles globaux dérivés des modèles GR [7] validés sur plus de 300 bassins versants. Il est donc apparu intéressant de transformer ce type de modèle global en un modèle distribué, ou plutôt semi-distribué en décomposant un bassin versant en sous-bassins. L'opération n'était pas anodine, car ce faisant on introduisait deux problèmes nouveaux :

- comment traiter les bassins intermédiaires ?

- quel modèle employer pour la composition des sous-bassins?

Pour commencer, il paraissait indispensable de préciser en quoi consistait cet avantage de la prise en compte de la diversité spatiale. Pour ce faire, nous nous sommes placés dans des conditions où les problèmes précédemment évoqués étaient réduits au minimum : les cas de confluence où une station de jaugeage existe sur chacune des branches en amont ainsi qu'à l'aval immédiat de la confluence. Dans ce cas idéal, il n'y a pas ou peu de bassins intermédiaires et le modèle d'intégration peut être assimilé à une simple addition des débits.

Deux analyses indépendantes ont ensuite été effectuées : (1) la comparaison des deux modélisations possibles que sont la modélisation globale et la modélisation distribuée et (2) la recherche des caractéristiques physiques permettant la différenciation de deux sous-bassins composant un bassin global. Cela nous a permis d'apprécier, d'une part, l'apport hydrologique d'une décomposition en sous-ensembles et d'autre part l'intérêt a priori d'une telle décomposition en fonction de contrastes physiques à identifier. La confrontation des résultats des deux études a été essentielle pour juger de l'intérêt d'une modélisation par rapport à l'autre (fig. ${ }^{\circ} 1$ ).

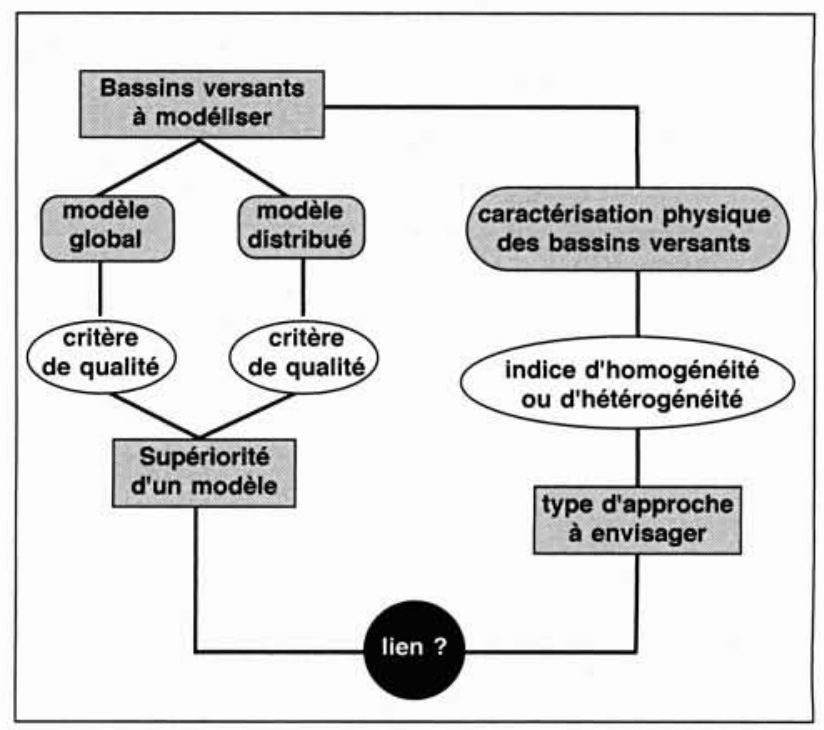

1. Schéma méthodologique. 


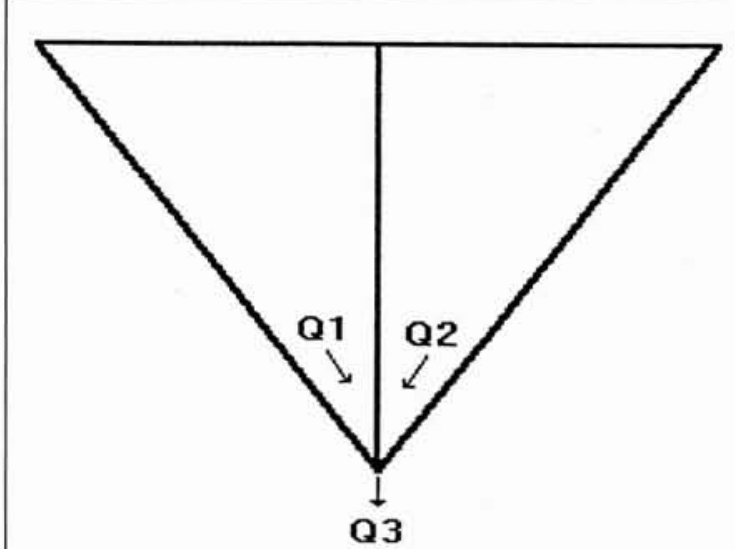

(Fig. 2a)

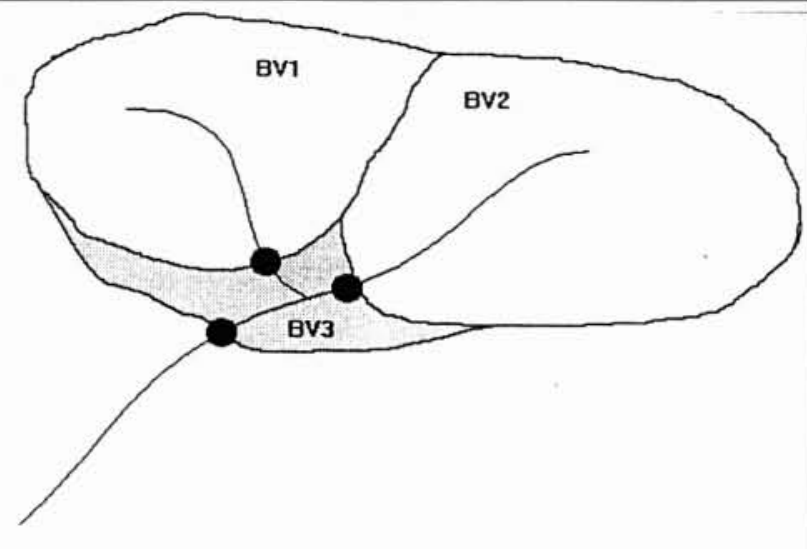

(Fig. 2b)

2. Découpage des bassins (fig. 2a (cas idéal), fig. 2b (cas réel)).

\subsection{Mode opératoire}

Le modèle semi-distribué utilisé est composé de la juxtaposition des deux modèles globaux relatifs à la partition du bassin global en deux sous-bassins comme l'indiquent les figures $2 \mathrm{a}$ et $2 \mathrm{~b}$.

Dans un souci de comparaison rigoureuse, la situation idéale est un découpage d'un bassin versant en deux entités égales, dont la somme correspond exactement au bassin initial (fig. 2a), mais trouver trois stations de jaugeage à proximité immédiate d'une confluence, avec une assez longue série de données communes est pratiquement impossible. Aussi le cas le plus fréquent réunit-il trois stations de jaugeage peu éloignées d'une confluence, ce qui introduit une donnée supplémentaire : le bassin intermédiaire, grisé sur la figure $2 \mathrm{~b}$, et dont il faut tenir compte dans la modélisation semi-distribuée.

En pratique, il y a très peu de situations présentant trois stations de jaugeage à proximité d'une confluence (figure $2 \mathrm{~b})$. Aussi, après une recherche minutieuse, région par région, cours d'eau par cours d'eau, en avons-nous recensé 15. La faiblesse de cet échantillon, qui représente pourtant la quasi-totalité des cas, nous a contraint à ne négliger aucune configuration (figure $n^{\circ} 3$ ).

\subsection{Les méthodes de comparaison}

Il existe dans la littérature un très grand nombre de critères indépendants du jugement de l'observateur, le plus classique étant celui des moindres carrés [8]. II est parfois commode de rendre cette quantité adimensionnelle pour faciliter les comparaisons ; c'est ce qui est fait avec le critère de NASH [1]. Le modèle est d'autant meilleur que le critère de Nash est proche de $100 \%$.

$$
N A S H=100 \times\left[1-\frac{\sum\left(Q_{0}-Q_{c}\right)^{2}}{\sum\left(Q_{0}-Q_{m}\right)^{2}}\right]
$$

avec $Q_{m}$ moyenne des débits observés $Q_{\mathrm{o}}$, et $Q_{c}$, débit calculé.

Par conséquent, en premier lieu, nous avons comparé les distributions statistiques des critères de Nash pour chaque option de modélisation, globale et semi-distribuée, et pour chaque bassin versant. En outre, dans la mesure du possible, les périodes d'étude ont été découpées en intervalles de quatre ans, et ceci pour deux raisons :

1/ Le faible nombre d'individus en notre possession nous incite à augmenter notre échantillon de $\mathrm{n}$ valeurs ;

2/ En découpant les périodes d'étude, on cale systématiquement le modèle sur plusieurs périodes et on le contrôle sur les autres, ce qui nous permet d'intégrer le caractère aléatoire des précipitations, et le risque de caler le modèle sur une période sèche, et de le contrôler sur une période humide, et réciproquement. Ainsi, sur une période d'étude de 20 ans, découpée en 5 intervalles, A, B, C, D, E, on obtient, in fine, 20 critères de qualité, correspondant aux périodes de calagecontrôle $\mathrm{AB}, \mathrm{AC}, \ldots, \mathrm{EC}, \mathrm{ED}$.

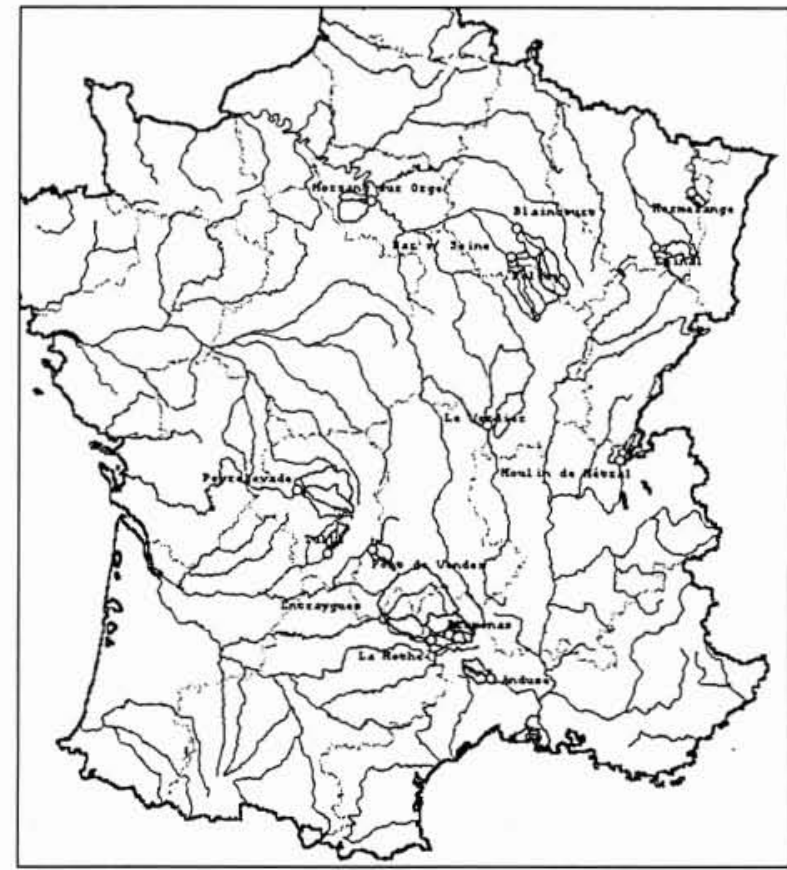

3 Situation géographique des bassins versants. 
Tableau 1. - Moyenne et variance des résultats de la modélisation globale et distribuée.

\begin{tabular}{|c|c|c|c|c|c|c|c|c|c|c|c|c|c|c|c|c|}
\hline Bassins & \multicolumn{2}{|c|}{ Aube } & \multicolumn{2}{|c|}{ Cantal } & \multicolumn{2}{|c|}{ Gard } & \multicolumn{2}{|c|}{ Corrèze } & \multicolumn{2}{|c|}{ Vienne } & \multicolumn{2}{|c|}{ Côte d'Or } & \multicolumn{2}{|c|}{ Lozère } & \multicolumn{2}{|c|}{ Aveyron } \\
\hline cours d'eau & \multicolumn{2}{|c|}{ Seine } & \multicolumn{2}{|c|}{ Sumène } & \multicolumn{2}{|c|}{ Gardon } & \multicolumn{2}{|c|}{ Corrèze } & \multicolumn{2}{|c|}{ Vienne } & \multicolumn{2}{|c|}{ Seine } & \multicolumn{2}{|c|}{ Lot } & \multicolumn{2}{|c|}{ Lot } \\
\hline critère en $\%$ & moy & var & moy & var & moy & var & moy & var & moy & var & moy & var & moy & var & moy & var \\
\hline Global & 89 & 12 & 84 & 12 & 84 & 30 & 91 & 6 & 89 & 2 & 91 & 17 & 86 & 8 & 64 & 77 \\
\hline Distrib & 89 & 4 & 85 & 7 & 87 & 11 & 91 & 7 & 89 & 1 & 91 & 12 & 85 & 9 & 66 & 28 \\
\hline Bassins & \multicolumn{2}{|c|}{ Ain } & \multicolumn{2}{|c|}{ Lozère 2} & \multicolumn{2}{|c|}{ Saône } & \multicolumn{2}{|c|}{ Moselle } & \multicolumn{2}{|c|}{ Essonne } & \multicolumn{2}{|c|}{ Aube 2} & \multicolumn{2}{|c|}{ Vosges } & \multicolumn{2}{|c|}{ SOMME } \\
\hline cours d'eau & \multicolumn{2}{|c|}{ Valserine } & \multicolumn{2}{|c|}{ Lot } & \multicolumn{2}{|c|}{ Arroux } & \multicolumn{2}{|c|}{ Sarre } & \multicolumn{2}{|c|}{ Orge } & \multicolumn{2}{|c|}{ Aube } & \multicolumn{2}{|c|}{ Moselle } & \multicolumn{2}{|c|}{ des BV } \\
\hline & moy & var & moy & var & moy & var & moy & var & moy & var & moy & var & moy & var & moy & var \\
\hline Global & 62 & 85 & 84 & 23 & 92 & 2 & 76 & 106 & 55 & 214 & 93 & 1 & 88 & 19 & 82 & 144 \\
\hline Distrib & 57 & 79 & 83 & 2 & 93 & 1 & 11 & 835 & 58 & 315 & 92 & 1 & 89 & 13 & 78 & 490 \\
\hline
\end{tabular}

\section{III — LES RÉSULTATS DE LA MODÉLISATION}

Chacun des 15 triplets de bassins a donc été modélisé, avec le modèle non linéaire GR4J [9], au pas de temps journalier, selon les approches globales et distribuées. GR4J [2, 10] est un modèle conceptuel pluie-débit à réservoirs, très robuste et simple à mettre en œuvre.

Le nuage de points défini par les couples de critères de Nash, pour les deux approches est présenté sur un graphe (figure $n^{\circ} 4$ ) situant en abscisse les valeurs obtenues pour l'approche globale, et en ordonnée, celles obtenues avec l'approche semi-distribuée ; la situation de ce nuage de point par rapport à la première bissectrice permet une première confrontation des deux approches. Si le nuage de points se trouve globalement au-dessus de la première bissectrice, l'approche semi-distribuée est meilleure, et si c'est l'inverse, l'approche globale est meilleure.

On remarque que les points sont agglomérés autour de la première bissectrice ; il n'y a donc pas de différence significative visuelle entre approche globale et semi-distribuée sauf dans deux cas :

- le cas de la Moselle située en zone périmontagneuse où les précipitations neigeuses sont importantes ; le calage par sous-bassin est peut-être plus délicat qu'un calage global ; l'approche globale est meilleure,

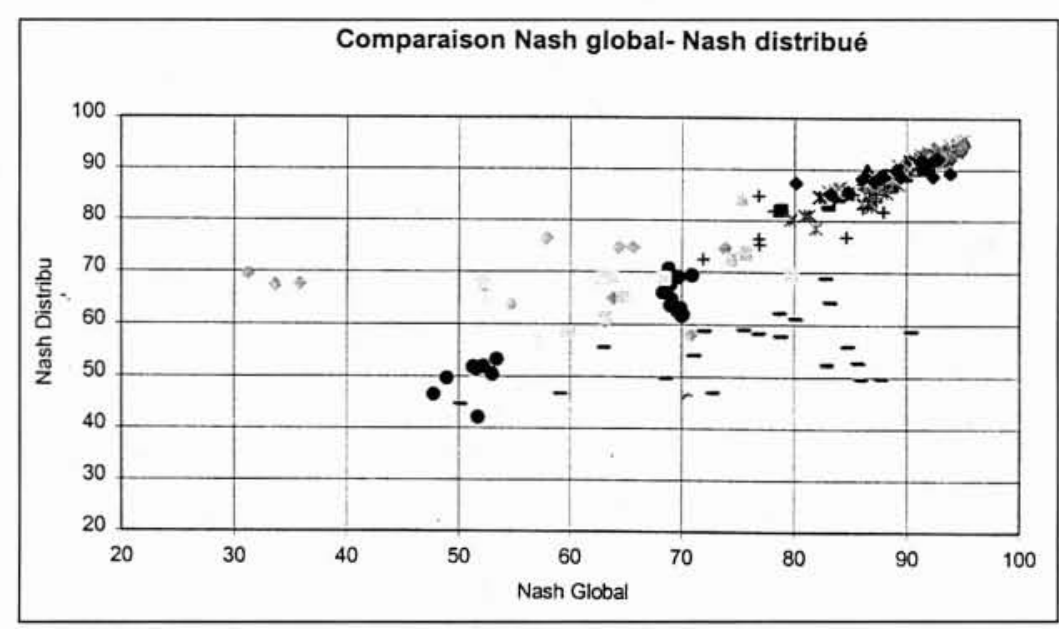

4. Comparaison des résultats de la modélisation globale et distribuée.
- le cas de l'Essonne possédant une rivière de nappe dans un de ses sous-bassin, l'approche semi-distribuée est meilleure.

Le tableau 1 donne les moyennes et variances des échantillons pour chaque bassin et chaque approche (critères de Nash obtenus pour chaque période de contrôle) :

Une rapide analyse de ce tableau montre que, si l'approche semi-distribuée ne montre pas des critères de Nash très supérieurs à ceux de l'approche globale, les variances sont souvent plus petites. Cela semble signifier que la partition d'un bassin versant entraîne une plus grande stabilité des résultats dans la modélisation pluie-débit.

Pour comparer les deux approches, il est aussi possible d'observer la distribution des critères de Nash par rapport à un quantile. Il faut pour cela tracer les graphes des fréquences au non dépassement pour chaque échantillon de critères de Nash, indépendamment de l'appartenance aux différents bassins, soit au total, pour chaque approche, 244 valeurs (figure $n^{\circ} 5$ ).

Tableau 2. - Valeurs du critère de Nash pour différents quantiles.

\begin{tabular}{|l|c|c|c|}
\hline \multicolumn{1}{|c|}{ Nash } & Quantile 0,1 & Quantile 0,3 & Quantile 0,5 \\
\hline Global & 63 & 83 & 88 \\
\hline Distribué & 58 & 81 & 88 \\
\hline
\end{tabular}

L'analyse du tableau $n^{\circ} 2$ et de la figure n 5 ne montre pas la supériorité d'une approche sur l'autre.

Pour une comparaison quantitative [11, 12], le test non paramétrique de Wilcoxon [13] a été utilisé afin de comparer les deux séries de critère de Nash pour chacune des deux approches.

Nous avons établi un classement entre les résultats de l'approche globale et ceux de l'approche semi-distribuée en fonction du test statistique T1 de Wilcoxon. Si celleci est négative, l'approche semi-distribuée est meilleure que l'approche globale, à condition que la valeur absolue de $\mathrm{T} 1$ soit plus grande que 1,64 (valeur correspondant à un seuil de signification de $5 \%$ ) ou supérieure à 1,28 (seuil de 10\%). 
Le tableau 3 montre qu'au seuil de signification $5 \%$ il existe une différence significative entre les approches globale et distribuée, pour les bassins AIN, MOSELLE et AUBE 2 où l'approche globale est meilleure et au seuil de $10 \%$ pour les bassins SAONE et GARD où l'approche semidistribuée est meilleure.

\section{IV — LIEN ENTRE DEGRÉ D'HÉTÉROGÉ- NÉITÉ DES BASSINS ET LEURS CARACTÉRISTIQUES PHYSIQUES}

L'écoulement à l'exutoire d'un bassin versant est déterminé premièrement par les conditions climatiques de la région, et deuxièmement par l'aménagement et l'occupation du territoire. Cependant, deux bassins voisins soumis aux mêmes conditions climatiques peuvent avoir un régime d'écoulement totalement différent. Cette différence est principalement causée par les diverses caractéristiques physiques des deux bassins comme le couvert végétal qui contrôle en partie l'évapotranspiration. C'est pourquoi, pour chaque bassin, nous avons recherché les différences et les similitudes des sous-bassins vis-à-vis de leurs caractéristiques physiques, du couvert végétal, de la pédologie et de la géologie (figure $n^{\circ} 6$ ).

Nous avons établi un protocole de comparaison pour déterminer l'homogénéité ou l'hétérogénéité d'un bassin visà-vis de ses deux sous-bassins.

DeCoursey [14] a mesuré les similitudes entre plusieurs groupes de bassins versants par la distance euclidienne :

$$
d_{j, k}=\left[\sum_{i=1}^{p}\left(x_{i, j}-x_{i, k}\right)^{2}\right]^{1 / 2}
$$

où $d_{j, k}$ adimensionnel, représente la distance entre les stations $j$ et $k, x_{i, j}$ est la $i^{\grave{e} m e}$ caractéristique de la station $j$ et $p$ est le nombre total de caractéristiques. Pour le couvert végétal, la topographie, la pédologie et la géologie les $x_{i}$ sont les valeurs attribuées en \% de la surface présente sur le bassin, aux formations pédolo-géologiques, au couvert végétal extrait de la base Corine Land Cover, et aux différentes classes d'altitude [15]. Les plus grandes similitudes sont ainsi liées aux plus petites distances. Ainsi, on peut calculer une distance pour tous les bassins versants, puis, en affectant un poids à chacune de ces distances, nous avons calculé un critère d'hétérogénéité pour chaque bassin.

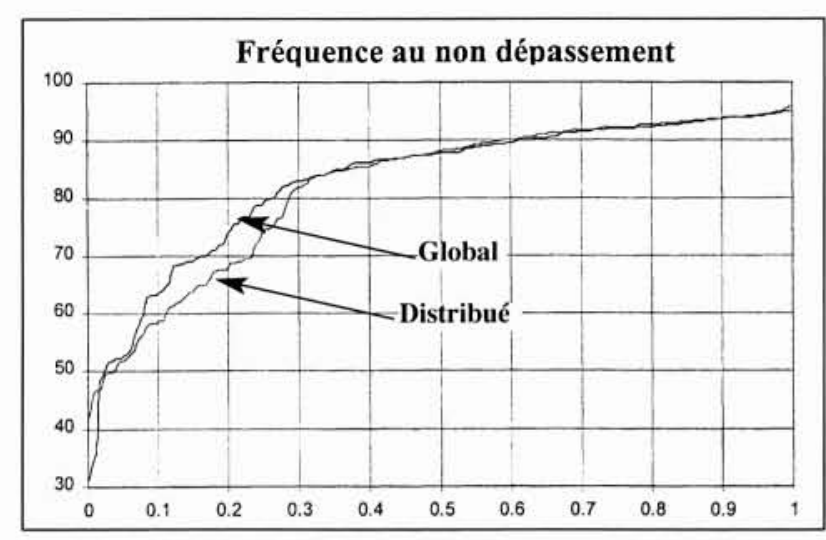

5. Distribution des critères de Nash.

Tableau 3. - Classement des résultats de l'approche globale et distribuée.

\begin{tabular}{|l|c|}
\hline Bassins & T1 de Wilcoxon \\
\hline SAONE & $\mathbf{- 1 , 4 1}$ \\
\hline GARD & $\mathbf{- 1 , 2 8}$ \\
\hline VIENNE & $-0,95$ \\
\hline AVEYRON & $-0,84$ \\
\hline VOSGES & $-0,80$ \\
\hline ESSONNE & $-0,69$ \\
\hline CANTAL & $-0,48$ \\
\hline COTE D'OR & $-0,08$ \\
\hline AUBE & 0,05 \\
\hline CORREZE & 0,06 \\
\hline LOZERE 2 & 1,18 \\
\hline LOZERE & 1,21 \\
\hline AIN & $\mathbf{2 , 3 8}$ \\
\hline AUBE 2 & $\mathbf{2 , 5 4}$ \\
\hline MOSELLE & $\mathbf{5 , 2 7}$ \\
\hline
\end{tabular}

$d_{1,2}=\sqrt{0.5 \times d_{\text {pedo }+ \text { geol }}^{2}+d_{\text {vegetal }}^{2}+0.5 \times d_{\text {topo }+ \text { hydro }}^{2}}$

Les résultats des distances entre les sous-bassins pour chaque domaine étudié et pour chaque bassin versant sont regroupés dans le tableau $n^{\circ} 4$ et comparés au classement effectué entre l'approche globale et distribuée par le test de Wilcoxon.

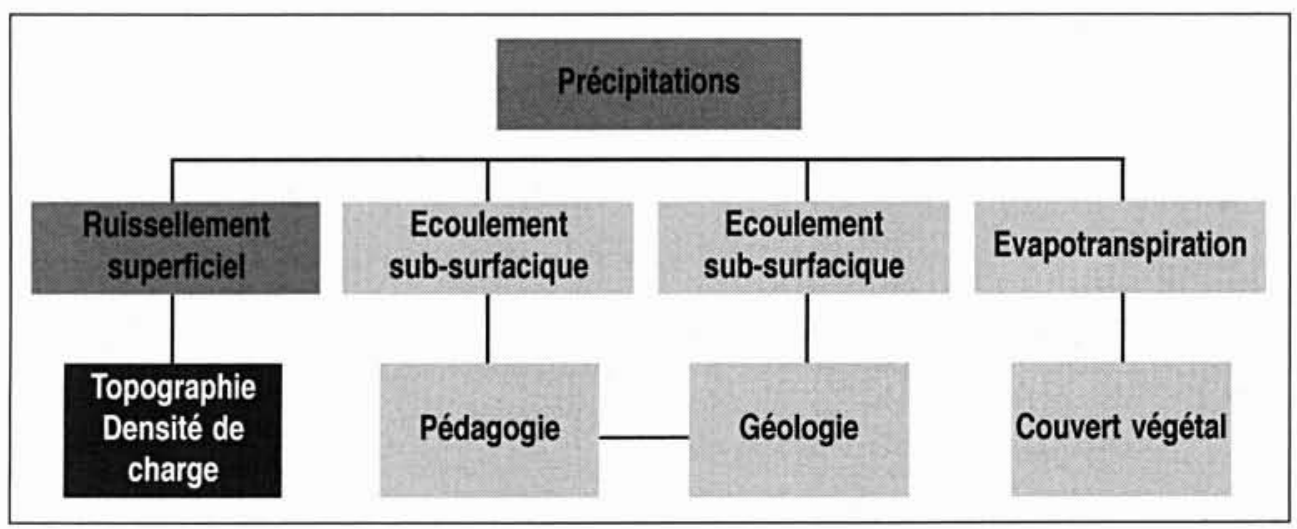

6. Choix des caractéristiques physiques. 
Tableau 4 - Classement des bassins par degré d'hétérogénéité et comparaison avec les résultats de la modélisation.

\begin{tabular}{|l|c|l|c|}
\hline \multicolumn{4}{|c|}{ T1 de Wilcoxon / Critère d'hétérogénéité } \\
\hline SAONE & $\mathbf{- 1 , 4 1}$ & CORREZE & $\mathbf{0 , 7 8}$ \\
\hline GARD & $\mathbf{- 1 , 2 8}$ & VOSGES & $\mathbf{0 , 7 4}$ \\
\hline VIENNE & $-0,95$ & AVEYRON & 0,50 \\
\hline AVEYRON & $-0,88$ & LOZERE2 & 0,42 \\
\hline VOSGES & $-0,80$ & VIENNE & 0,40 \\
\hline ESSONNE & $-0,69$ & SAONE & 0,39 \\
\hline CANTAL & $-0,48$ & GARD & 0,37 \\
\hline COTE D'OR & $-0,08$ & LOZERE & 0,35 \\
\hline AUBE & 0,05 & CANTAL & 0,34 \\
\hline CORREZE & 0,06 & AIN & $\mathbf{0 , 2 4}$ \\
\hline LOZERE2 & 1,18 & MOSELLE & $\mathbf{0 , 2 4}$ \\
\hline LOZERE & 1,21 & ESSONNE & $\mathbf{0 , 2 2}$ \\
\hline AIN & $\mathbf{2 , 3 8}$ & COTE D'OR & $\mathbf{0 , 2 1}$ \\
\hline AUBE 2 & $\mathbf{2 , 5 4}$ & AUBE & $\mathbf{0 , 1 9}$ \\
\hline MOSELLE & $\mathbf{5 , 2 7}$ & AUBE 2 & $\mathbf{0 , 1 5}$ \\
\hline
\end{tabular}

Dans ce tableau, plus la distance est faible plus les caractéristiques entre sous-bassins sont semblables et plus le bassin est homogène et inversement. Ce tableau montre que nous pouvons regrouper les bassins versants en trois groupes : les bassins très hétérogènes, les bassins hétérogènes et les bassins homogènes, mais ne montre pas un recoupement entre les classements pour chacune des deux comparaisons.

La représentation graphique de ces deux classifications est présentée sur la figure $n^{\circ} 7$ avec en abscisse les valeurs du critère d'hétérogénéité, et en ordonnée celles de la variable de Wilcoxon pour chaque bassin.
Au regard des résultats, il n'est pas immédiat, de mettre en évidence un lien entre les variables explicatives retenues et les résultats de la modélisation, avec le jeu de données dont nous disposons.

Les 15 triplets de bassins versants étudiés ont donc été caractérisés par trois grands paramètres : (i) leur superficie, (ii) leur variabilité hydrologique intra-bassin à l'aide du test non paramétrique de Wilcoxon appliqué à la comparaison des résultats des approches globale et semi-distribuée et (iii) leur degré d'hétérogénéité défini plus haut par intégration de leur composition géologique, pédologique, topographique, et végétale.

L'analyse statistique de ces résultats révèle tout d'abord que les critères de variabilité hydrologique ainsi que les degrés d'hétérogénéité des bassins versants n'obéissent apparemment pas à des lois d'échelle. Néanmoins, il est très probable que cela soit dû au trop faible nombre de bassins étudiés. En effet, si l'on classe les bassins en trois familles de taille $\left(200\right.$ à $600 \mathrm{~km}^{2}, 900$ à $1600 \mathrm{~km}^{2}$ et 2300 à $3200 \mathrm{~km}^{2}$ ), on observe une tendance montrant que la moyenne des valeurs T1 de Wilcoxon, et leur écart-type, calculés par famille de taille de bassin, diminuent en fonction de la superficie moyenne du bassin versant (tableau $n^{\circ} 5$ et figure $n^{\circ} 8$ ).

Cela tend à révéler (i) que plus le bassin est grand, plus l'approche semi-distribuée serait recommandée. Cela serait attribuable au fait que le nombre d'unités du milieu type (unités géologiques, pédologiques, topographiques ou végétales) a tendance à augmenter avec la taille d'un bassin, rendant plus complexe son analyse et (ii) que lorsque la taille du bassin est trop petite, le doute tendrait à s'installer quant au choix d'une approche globale ou d'une approche semi-distribuée. On rejoindrait là la notion de bassin versant représentatif (taille représentative) qu'il conviendrait donc de préciser.

Une analyse discriminante révèle ensuite que le degré d'hétérogénéité des bassins, basé sur leurs déterminants hydrologiques, est le facteur le plus discriminant. Celui-ci permet de classer les bassins étudiés en trois familles significativement très différentes ( $98 \%$ de probabilité) : les bassins très hétéro-

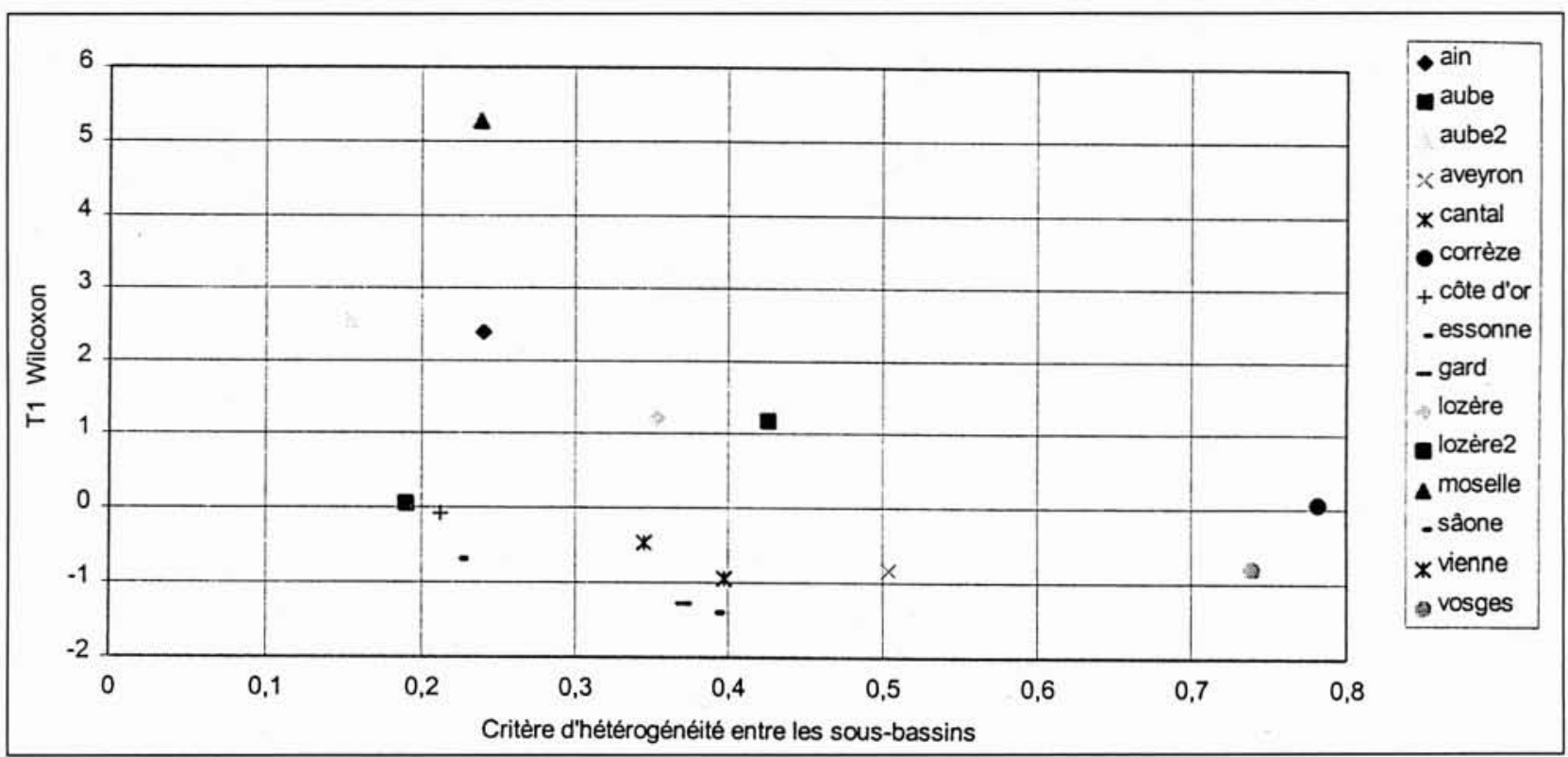

7. Classification des bassins par le critère d'hétérogénéité et le test de Wilcoxon. 


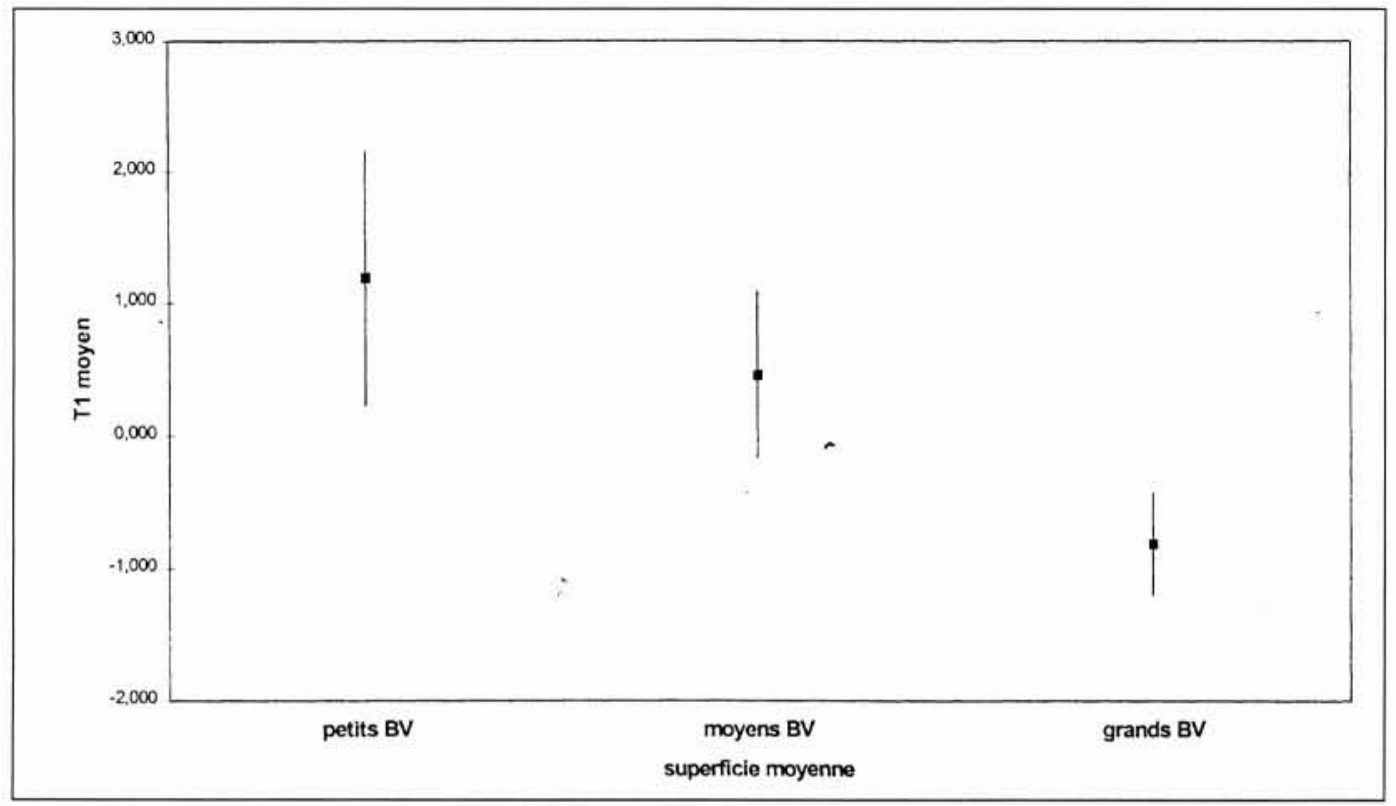

8. Classement des bassins par taille et $\mathrm{T} 1$ de Wilcoxon moyen par classe. gènes (Corrèze, Vosges), les bassins hétérogènes (Aveyron, Lozère 2, Vienne, Saône, Gard, Lozère, Cantal) et les bassins homogènes (Ain, Moselle, Essonne, Côte d'Or, Aube, Aube 2).

Une analyse de variance permet de mettre en évidence une tendance : les bassins hétérogènes se différencient, à $80 \%$ de probabilité, des bassins homogènes par leur valeur $\mathrm{T} 1$ de Wilcoxon (tableau $n^{\circ} 6$ ).

L'approche semi-distribuée serait donc plutôt recommandée pour les bassins hétérogènes alors que c'est l'approche globale qui serait recommandée dans le cas des bassins homogènes.

Toutefois, la faible taille de notre échantillon ne permet pas d'apporter une réponse concluante à cette étude. Ces résultats montrent cependant que cette thématique est pro- metteuse et qu'il faudrait, si possible, l'étendre à un nombre plus important de bassins.

\section{D CONCLUSION ET PERSPECTIVES}

En travaillant sur le maximum de triplets de bassins possible, soit quinze au total, et sur des périodes suffisamment longues, l'étude statistique des critères de Nash, avec l'utilisation du test non paramétrique de Wilcoxon, n'a pas permis de mettre en évidence une différence nette entre une modélisation globale et une modélisation distribuée. Mais lorsque I'on classe les bassins en fonction de leur superficie, on observe une tendance montrant l'intérêt d'utiliser l'approche semi-distribuée lorsque la taille du bassin augmente. Certes,

Tableau 5. - Classement des bassins par taille et calcul du TI moyen par classe.

\begin{tabular}{|c|c|c|c|c|c|}
\hline \multicolumn{2}{|c|}{ Petits bassins } & \multicolumn{2}{|c|}{ Bassins moyens } & \multicolumn{2}{|c|}{ Grands bassins } \\
\hline Bassins & Superficie & Bassins & Superficie & Bassins & Superficie \\
\hline Gard & 546 & Essone & 922 & Vienne & 2296 \\
\hline Lozère & 465 & Lozère 2 & 1164 & Côte d'or & 2340 \\
\hline Cantal & 401 & Vosges & 1219 & Saone & 3166 \\
\hline Ain & 395 & Aube & 1450 & Aveyron & 5460 \\
\hline Corrèze & 356 & Aube2 & 1640 & & \\
\hline Moselle & 192 & & & & \\
\hline $\begin{array}{l}\text { Moyenne du T1 } \\
\text { de Wilcoxon }\end{array}$ & 1,19 & $\begin{array}{l}\text { Moyenne du T1 } \\
\text { de Wilcoxon }\end{array}$ & 0,46 & $\begin{array}{l}\text { Moyenne du T1 } \\
\text { de Wilcoxon }\end{array}$ & $-0,81$ \\
\hline écart -type & 0,97 & écart-type & 0,63 & écart-type & 0,39 \\
\hline
\end{tabular}

Tableau 6. - Classement des bassins par degré d'hétérogénéité et calcul du TI moyen par classe.

\begin{tabular}{|c|c|c|}
\hline Bassins & moyenne du T1 de Wilcoxon & écart- type \\
\hline hétérogènes & $-0,29$ & 0,48 \\
\hline homogènes & 1,58 & 0,92 \\
\hline \multicolumn{2}{|c|}{ test de Fisher à $80 \%$ de probabilité : 1,844} \\
\hline
\end{tabular}


l'échantillon de base est assez réduit. Néanmoins, il représente la quasi-totalité des bassins versants français présentant la particularité de réunir trois stations de jaugeage à proximité d'une confluence. Pour aller plus avant, il faudra désormais se tourner vers les pays limitrophes pour rechercher des conditions similaires, en gardant un esprit critique vis-à-vis des données qui doivent rester homogènes quelle que soit leur origine. Ensuite, nous avons voulu voir s'il y avait une correspondance entre l'hétérogénéité caractérisant les deux sous-bassins d'un même triplet et les différences de résultats apparaissant entre les deux approches de modélisation. Pour cela, nous avons élaboré une méthode de comparaison basée sur une combinaison des distances euclidiennes entre les éléments caractéristiques de chaque sous-bassin à l'intérieur de plusieurs domaines d'étude : pédologie, géologie, couvert végétal, topographie et densité de drainage. Notre méthode de comparaison a consisté à établir un parallèle entre un classement des bassins selon leurs différences et un autre selon le degré de supériorité de l'approche distribuée par rapport à l'approche globale. Malgré le nombre trop faible de triplets de bassins, une tendance a pu être mise en évidence montrant que plus le bassin est hétérogène, plus l'approche semi-distribuée est recommandée. Ces résultats doivent cependant être confortés par un échantillonnage plus large de bassins.

\section{Références}

[1] NASH, J. E., SutcurfE, J. V.. 1970. - River flow forecasting through conceptual models. J. Hydrol., n¹0, pp 282-290.

[2] Michel. C., Eduatno, Lebandier T, 1991, - Progrès et application de la modélisation conceptuelle pluie-débit. Rapport quadriennal du CNFGG 1987-1990, Section VI : Sciences Hydrologiques, pp 219-222.
[3] MaKhi.ouf Z., 1994. - Compléments sur le modèle pluie-débit GR4J et essai d'estimation de ses paramètres. Thèse de doctorat, $426+208$ p. Université Paris XI Orsay.

[4] Beven K., 1989. - Changing ideas in hydrology-the case of physically based models. Journal of hydrology, $\mathrm{n}^{\circ} 105$, pp 157-172.

[5] Nathan R. J., Mc MaHON T. A., 1990. - Identification of homogeneous regions for the purposes of regionalisation. Journal of Hydrology, $n^{\circ} 1-4$, pp 217-238.

[6] Aввотt M. B., Refsgando J. C., 1996. - Distributed Hydrological Modelling. Water Science and Technology Library, Kluwer Academic Publishers.

[7] MaKhlouf Z., Michel. C., 1992. - Relation entre les paramètres d'un modèle global pluie-débit et les caractéristiques physiques d'un bassin versant breton. 8ème Journées Hydrologiques de l'ORSTOM, 22/23 sept. 1992.

[8] MARTINEC J., RANGo A., 1989, - Merits of statistical criteria for the performance of hydrological models. Water Resources Bull. USA, $n^{\circ} 25, \mathrm{pp}$ $421-432$.

[9] Eduatno, Miches. C., 1989. - Un modèle pluie-débit journalier à trois paramètres. La Houille Blanche, $n^{\circ} 2$, pp 113-121.

[10] NaCimento DE Ouveira N, 1995. - Appréciation à l'aide d'un modèle empirique des effets d'actions anthropiques sur la relation pluie-débit à l'échelle d'un bassin versant. Thèse de doctorat del'ENPC. 550p.

[11] Dodge Y., 1993, - Statistique, Dictionnaire encyclopédique. Dunod, Paris. 408 p.

[12] GiBBons J.D., 1971. - Nonparametric Statistical Inference. McGrawHill, Kogakusha, Tokyo,

[13] WILcoxon F., 1945, - Individual comparisons by ranking methods. Biometrics, $\mathrm{n}^{\circ} 1$, pp 80-83.

[14] Decoursey D.G., 1973, - Objective regionalization of peak flow rates. Water resources publications, pp 395-405.

[15] BAUDEZ J.C., 1997. - Détérminants hydrologiques régionaux pour la gestion et la prévision des ressources en eau. Mémoire de DEA. Paris VI. $88 \mathrm{p}$. 